\title{
EDITORIAL
}

\section{Magnetic Resonance Imaging and Spectroscopy: Present and Future}

Magnetic Resonance Imaging (MRI) demonstrates abnormalities of the brain and the intracranial course of the cranial nerves with remarkable clarity. The advantages and disadvantages of the technique are now well established and the role in neuro-opthalmology is discussed in the current issue. Unfortunately the purchase, installation and running of units are very expensive and are likely to be in short supply for many years: it is only recently that Computerised Tomography (CT) has become widely available outside the USA and Japan. In an ideal world all intracranial neuro-ophthalmological problems should be examined by MRI but unfortunately a highly critical and selective approach has to be made. The unit at Cambridge, which commits $60 \%$ of the available time to the neuraxis, only accepts chiasmal compression prior to surgery as an absolute indication-angiography is no longer used to delineate the carotids.

It is difficult to envisage that MRI will become widely available until the cost is closer to CT. The enormous initial, and continuing, capital investment in research and development by the manufacturers along with the cost of helium, a finite resource that is relatively cheap in the UK, and service contracts will continue to keep costs at a prohibitive level especially for the District Hospital unless there is substantial private income. Less expensive capital and revenue units are available with low and ultra low field strength using resistive and permanent magnets. It is generally considered that high field systems are 'best' for examining the nervous system and there is no doubt that with the high signal to noise they produce aesthetic images. However there is no evidence to indicate a greater clinical usefulness than the low field systems. A large pituitary tumour, its extent and relationship to the carotid arteries can be equally well shown on any modern system. A high field instrument using thinner sections can demonstrate small areas of altered signal intensity in the pituitary which may or may not be microadenomas (surgery and imaging do not always agree). Ten years of high resolution CT with substantial doses of contrast medium have failed to resolve this problem. The arguments for and against an optimal field strength are less intense now and in the UK the 'optimal field strength is that which you have' (though high field ( $1.5 \mathrm{~T}$ or greater) is required for spectroscopy.

One of the driving forces behind the development of Magnetic Resonance is the possibility of tissue specificity using relaxation data and spectroscopy from the laboratory. Initially it was suggested that malignant tissue had a distinct $\mathrm{T} 1$ and $\mathrm{T} 2$ relaxation signature. Unfortunately, at least in brain tumours, neither T1 nor T2 alone or in combination, provide any specific diagnostic value. In vitro very small changes in protein concentration are reflected in the relaxation parameters but in vivo only gross changes are detected. The generally improved delineation of size, shape and position often helps to narrow the differential diagnosis but the final common pathway of injury to nervous system tissue, as detected by Magnetic Resonance, is the increase in free water and the ubiquitous high signal on T2 weighted images sequences. Spectroscopy is dependent on the property of identical nuclei having slightly different resonance frequencies depending on their chemical environment. Protons in water have a different frequency to those in fat giving rise to the familiar chemical shift artefact in imaging. In the brain $\mathrm{N}$-acetylaspartate, $\gamma$-amino-butyrate, glutamine, glutamate, aspartate, creatine and phosphocreatine, choline-containing compounds, taurine and inositols can be detected and quantified by proton spectroscopy. This is a technically very demanding procedure and difficult, as yet, to integrate into everyday clinical imaging. In addition, localisation of the spectra is coarse requiring volumes in the order of $27 \mathrm{cc}$. There are differences between meningiomas and gliomas but the size of the lesions are such that the diagnosis is evident from ordinary imaging. High resolution spectra from the eye in vitro are easily obtainable but localised spectra from a small mobile structure is difficult.

Initially one of the usually stated advantages of MRI was the high contrast sensitivity obviating the use of artificial, and potentially toxic, contrast agents as used extensively in CT. The use of Gadolinium-DPTA shows the breakdown of the blood brain barrier and can be useful in separating areas of tumour from the surrounding oedema and detecting both en plaque and optic nerve meningiomas. Its use in assessing the age of Multiple Sclerosis plaques is a most interesting research tool but is difficult to justify in routine clinical practice as it changes the non-invasive nature of the examination and costs $£ 63$ (at current UK prices) without, as yet, definite therapeutic and prognostic implications. It would be easy to recommend its use in a wider group of patients on the grounds that it might help but on the other hand it may well be equally as profitable to spend the time with extra imaging sequences tailored to the particular clinical problem.

The orbit and its contents are so well demonstrated by CT, with the natural contrast from intra orbital fat, that the role of MRI is not fully evolved. It is unsuitable, or contra-indicated, in the common problems of blow out fractures, thyroid ophthalmopathy, and foreign body detection and localisation. MRI is valuable however where coronal sections on CT cannot be obtained, assessing the intracranial extent of an orbital mass and where multiple follow up examinations are likely to be required.

In spite of the problems MRI is the imaging technique of the future. Costs and imaging time will be reduced and dedicated machines will be developed. The instruments of today are leviathans: large, slow, and doomed for extinction. Imagine a small unit dedicated to the orbit with fast 3 dimensional data acquisition so that the image can be rotated in real time and individual areas examined in more detail: perhaps the biochemistry will be as available as plasma proteins are today.

C.E.L. Freer, Department of Radiology, MRIS Unit, Addenbrooke's Hospital, Cambridge 


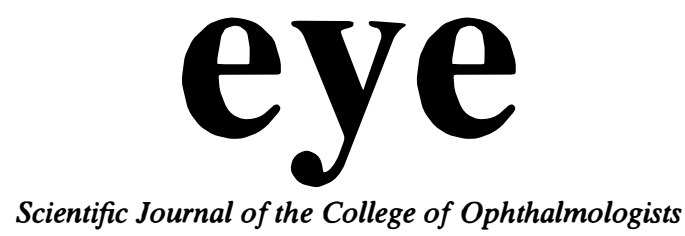

1989

\section{VOLUME 3 - Part 6}

Editor

P. G. Watson FRCS 